RESEARCH ARTICLE

\title{
Uterine Rupture during Subsequent Pregnancy following Adeno- myomectomy - Report of Five Cases and Proposal for Prevention
}

\section{Masato Nishida ${ }^{1}$, Yasuo Otsubo ${ }^{1}$, Yuko Arai ${ }^{1}$, Ryota Ichikawa ${ }^{1}$, Yuzuru Kondo ${ }^{2}$, Hiroya Itagaki ${ }^{1}$ and Miyako Sakanaka}

${ }^{1}$ Department of Obstetrics and Gynecology, National Hospital Organization, Kasumigaura Medical Center, Japan

${ }^{2}$ Department of Pathology, National Hospital Organization, Kasumigaura Medical Center, Tsuchiura, Japan

*Corresponding author: Masato Nishida, Department of Obstetrics and Gynecology, National Hospital Organization, Kasumigaura Medical Center, 2-7-14 Shimotakatsu, Tsuchiura, Ibaraki, 300-8585, Japan, Tel: +81-29-822-5050, Fax:+81-29-824-0494, E-mail: nishidamasato@mac.com

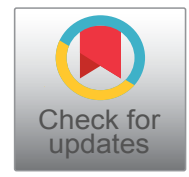

\begin{abstract}
Purpose: The risk of uterine rupture is a major concern for women who become pregnant after undergoing an adenomyomectomy. The aim of this study was to investigate the mechanism of uterine rupture and improve the surgical procedure used for prevention.

Material and methods: Five patients who experienced uterine rupture during subsequent pregnancy after undergoing an adenomyomectomy performed with an open laparotomy were retrospectively investigated.

Results: In all cases, the uterine rupture lesion occurred on the suture mark of the myometrium and uterine cavity. Placenta percreta was confirmed in all patients during surgery, while histological findings revealed chorionic villi in resected myometrium specimens obtained form 2 .

Conclusion: A uterine rupture during subsequent pregnancy following an adenomyomectomy may be related to placenta increta or percreta.
\end{abstract}

\section{Keywords}

Adenomyosis, Conservative surgery, Uterine rupture, Pregnancy

\section{Introduction}

Adenomyosis can occur in women of active reproductive age [1], who often wish to preserve their uterus, though the standard treatment generally selected is hysterectomy. The procedure used for uterine-sparing surgery for adenomyosis is an adenomyomectomy which is performed for relief of dysmenorrhea and/or hypermenorrhea, prevention of recurrence, and re- taining the possibility of conception following surgery $[2,3]$. Various perinatal complications are associated with an adenomyomectomy [4], thus consensus for its indications among obstetricians has not been obtained. Notably, uterine rupture is a lethal condition for both mother and fetus [5].

It is clinically important to investigate the mechanism of uterine rupture in affected patients, and also to take precautions and/or seek to improve the surgical procedure used for preventing uterine rupture in those who achieve pregnancy after undergoing an adenomyomectomy. Here, we present 5 cases of uterine rupture during pregnancy and discuss possible techniques for its prevention.

\section{Materials and Methods}

Since March 2002, we have performed conservative surgical treatment for 1603 women with uterine adenomyosis (focal, $n=1200$; diffuse, $n=403$ ) who were diagnosed based on magnetic resonance imaging (MRI) findings [6]. Uterine adenomyosis can be classified into 2 categories; focal adenomyosis, with lesions localized in the specific uterine wall, and diffuse adenomyosis, with lesions involving the entire uterus [7]. The surgical procedures employed at our institution consist of 2 different methods. For focal type adenomyosis, excision of the lesion including surface serosa is performed using a high-frequency cutter equipped with a round type of loop electrode, after which the muscle layer is re-

Citation: Nishida M, Otsubo Y, Arai Y, Ichikawa R, Kondo Y, et al. (2018) Uterine Rupture during Subsequent Pregnancy following Adenomyomectomy - Report of Five Cases and Proposal for Prevention. Int J Womens Health Wellness 4:070. doi.org/10.23937/2474-1353/1510070 Received: May 03, 2018: Accepted: May 17, 2018: Published: May 19, 2018

Copyright: (c) 2018 Nishida M, et al. This is an open-access article distributed under the terms of the Creative Commons Attribution License, which permits unrestricted use, distribution, and reproduction in any medium, provided the original author and source are credited. 
constructed by suturing (Type I procedure). For diffuse type, following longitudinal and asymmetrical dissection of the uterus with a high-frequency electrical surgical knife, along with preservation of the uterine cavity, the adenomyosis lesion is excised from the incision area site using a loop electrode attached to a high-frequency cutter, after which the uterus is rejoined (Type II procedure) $[2,3]$. Both procedures are performed with an open laparotomy approach.

We retrospectively investigated 5 patients who achieved pregnancy after undergoing an adenomyomectomy at our institution and experienced uterine rupture. Frequency, type of adenomyosis, surgical method, location of placentation, clinical course of pregnancy, period of onset of uterine rupture, symptoms at time of occurrence, and laparotomy and pathological findings were analyzed.

\section{Results}

At our institution, we have performed conservative surgical treatment for at total of 1603 women with uterine adenomyosis. Following that procedure, 304 pregnancies occurred in 244 of our patients (focal type, $n$ = 200; diffuse type, $n=44)$. Of those 244 patients, 136 $(54.5 \%)$ proceeded to delivery, $32(13.1 \%)$ were pregnant at the time of writing, while $5(2.0 \%)$ experienced a uterine rupture and $2(0.8 \%)$ had pregnancy loss due to uterine rupture. The median resected adenomyosis lesion weight in the proceed to delivery and uterine rupture groups was $128.7 \mathrm{~g}$ (1-720 g) and $128.4 \mathrm{~g}$ (76-176 g), respectively. Additional analysis performed to compare the sizes of the resected adenomyosis lesions revealed no significant relationships between the groups.

\section{Clinical features}

Outcomes and other characteristics of all cases of uterine rupture at our institution are shown in Table 1.
Details of those 5 cases are presented following.

Case 1: A 38-year-old gravida 2 nulliparous patient who wished to become pregnant first came to us in 2007 due to dysmenorrhea with recurrent adenomyosis following a previous cystectomy performed at the age of 32 years and an adenomyomectomy performed at 35-years-old at a different institution. The visual analogue scale was 9.7 and the level of CA125 was elevated to $130 \mathrm{IU} / \mathrm{ml}$. An enlarged uterus, similar to that seen after 12 weeks of pregnancy, was found, while ultrasound imaging and MRI findings revealed adenomyosis form the uterine fundus to posterior uterine wall, with normal ovaries. We performed a Type I procedure and the total weight of the excised adenomyosis was $152 \mathrm{~g}$. Nine months after surgery, she conceived with the aid of in vitro fertilization embryo transfer (IVF-ET), though later miscarried. Sixteen months after surgery, she conceived again with IVF-ET. The pregnancy was normal until 31 weeks of gestation, at which time sudden severe abdominal pain occurred requiring an immediate caesarean delivery followed by a hysterectomy at another institution. The post-operative diagnosis was placenta increta with uterine rupture. The neonate survived.

Case 2: A 35-year-old gravida 0 patient came to us in 2008 for infertility lasting 10 years, as well as a known left single kidney and left hemi-uterus. MRI findings revealed that the right rudimentary cavity had been replaced with adenomyosis. Conservative surgical management was selected, as follows. After removing the right rudimentary cavity, excision of the lesion on the left hemi-uterus that was affected by adenomyosis, including the surface serosa, was performed using a high-frequency cutter equipped with a round type of loop electrode. Next, a uterine-plasty for the hemi-uterus [8] was performed, after which the muscle layer was

Table 1: Characteristics of 5 uterine rupture cases.

\begin{tabular}{|c|c|c|c|c|c|}
\hline Case \# & 1 & 2 & 3 & 4 & 5 \\
\hline Age (year) & 38 & 35 & 31 & 34 & 32 \\
\hline Paritya & $0-0-2-0$ & $0-0-0-0$ & $0-0-0-0$ & $0-0-0-0$ & $0-0-0-0$ \\
\hline Past history \& complication & $\begin{array}{l}\text { Myomectomy \& } \\
\text { adenomyomectomy }\end{array}$ & Left hemi-uterus & Myomectomy & Adenomyomectomy & None \\
\hline Location of adenomyosis & $\begin{array}{l}\text { Fundus \& posterior } \\
\text { wall }\end{array}$ & $\begin{array}{l}\text { Rudimentary cavity } \\
\text { \& right wall }\end{array}$ & Posterior wall & Diffuse & Diffuse \\
\hline $\begin{array}{l}\text { Resected tissue of } \\
\text { adenomyosis }(\mathrm{g})\end{array}$ & 152 & 178 & 76 & 80 & 158 \\
\hline $\begin{array}{l}\text { Procedure of } \\
\text { adenomyomectomy }\end{array}$ & Type I & Type I & Type I & Type II & Type II \\
\hline Conceive mode ${ }^{b}$ & IVF-ET & $\mathrm{Sp}$ & IVF-ET & $\mathrm{Sp}$ & IVF-ET \\
\hline $\begin{array}{l}\text { Gestational age of } \\
\text { uterine rupture (weeks) }\end{array}$ & 31 & 27 & 30 & 16 & 19 \\
\hline Placentation & Posterior & Unknown & Posterior & Fundus & Fundus \\
\hline $\begin{array}{l}\text { Symptom of uterine } \\
\text { rupture }\end{array}$ & $\begin{array}{l}\text { Acute abdominal } \\
\text { pain }\end{array}$ & $\begin{array}{l}\text { Acute abdominal } \\
\text { pain }\end{array}$ & $\begin{array}{l}\text { Acute abdominal } \\
\text { pain }\end{array}$ & $\begin{array}{l}\text { Acute abdominal } \\
\text { pain }\end{array}$ & Epigastralgia \\
\hline Therapy for uterine rupture & $\begin{array}{l}\text { Cesarean section \& } \\
\text { hysterectomy }\end{array}$ & Cesarean section & $\begin{array}{l}\text { Cesarean section } \\
\text { \& metroplasty }\end{array}$ & Metroplasty & Metroplasty \\
\hline Surgery diagnosis & Placenta increta & Uterine rupture & Placenta percreta & Placenta percreta & Placenta percreta \\
\hline
\end{tabular}

aFirst: figure term delivery; second: premature delivery; third: abortion; fourth: means a liveborn infant; ' embryo (or blastocyst) transfer; $\mathrm{Sp}$ : spontaneous conception. 


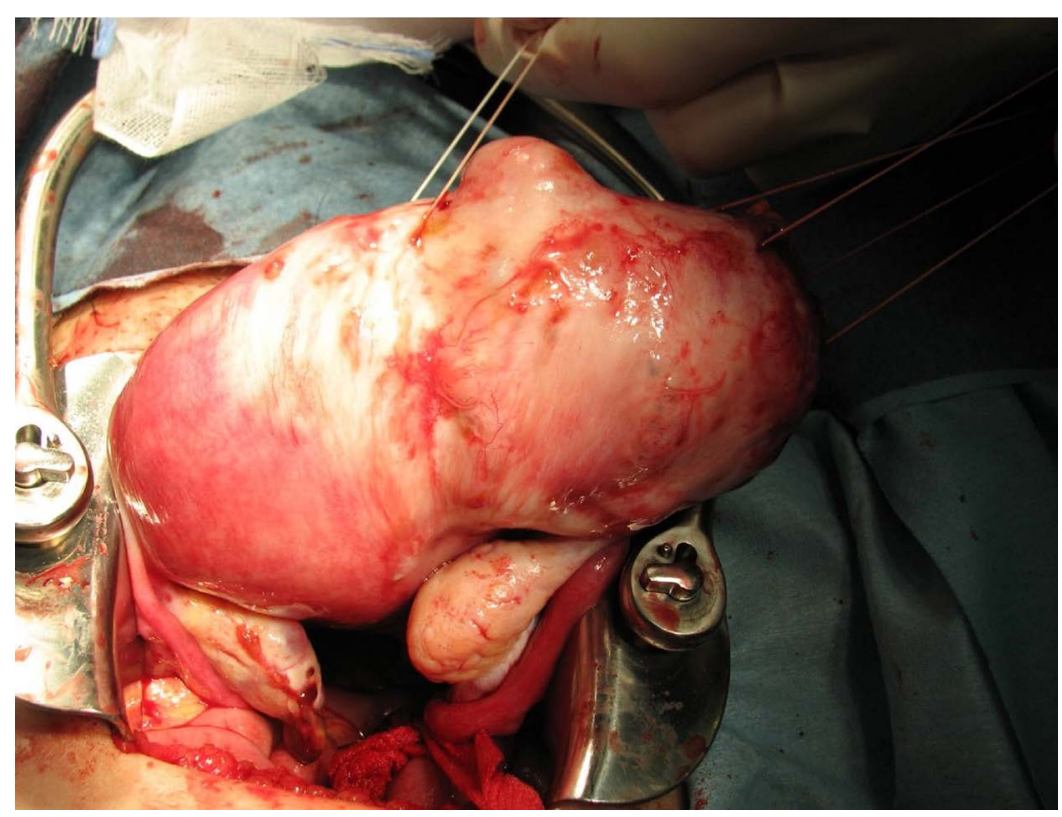

Figure 1: Appearance of uterus in Case 2 when abdomen was opened.

A hypertrophic myometrium with adenomyosis was observed in the right uterine rudimentary cavity.

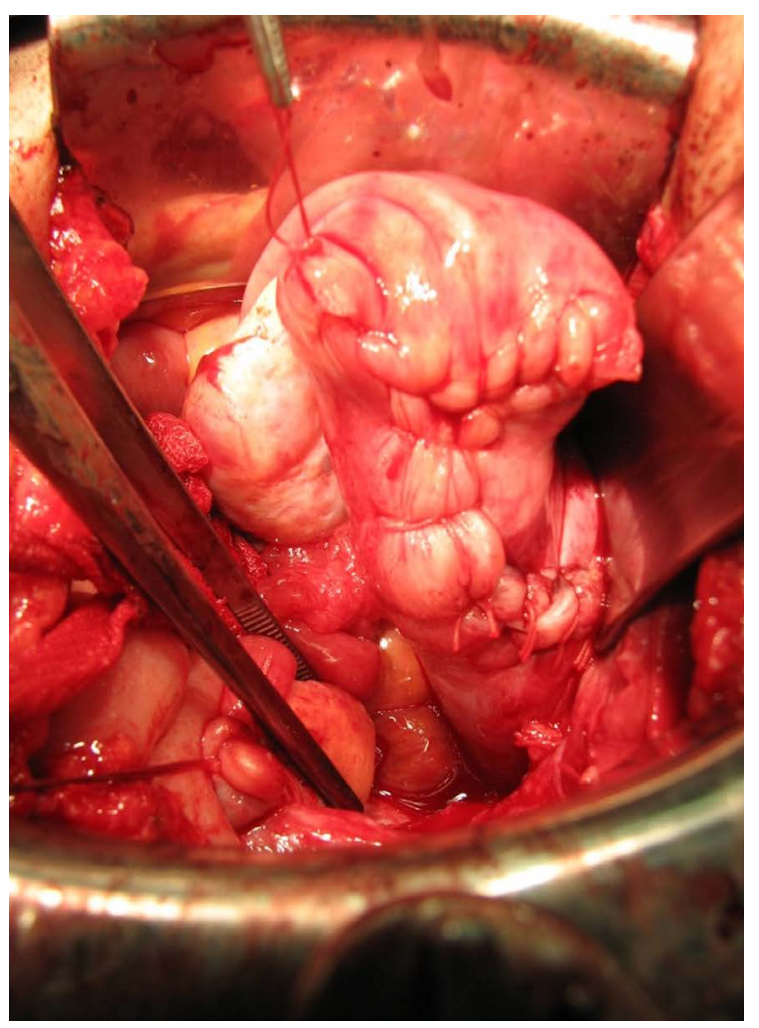

Figure 2: Appearance of reconstructed uterus in Case 2. Uterine myometrium received a Y-shaped suture was observed.

reconstructed using a Type I procedure (Figure 1 and Figure 2). The total weight of excised tissue was $176 \mathrm{~g}$. Three months after surgery, the patient spontaneously conceived and achieved a normal pregnancy. However, at 27 weeks of gestation, she experienced sudden abdominal pain with intraperitoneal bleeding, which required an immediate caesarean delivery performed at another institution. Bleeding from the uterine defect wall within a space of several millimeters was confirmed during surgery. The neonate weighed $1106 \mathrm{~g}$ and survived.
Case 3: A 31-year-old gravida 0 patient wished to become pregnant and was referred to our hospital in 2009 due to recurrent adenomyosis following a previous cystectomy procedure for bilateral ovarian endometriotic cysts and myomectomy at 29 years performed at another institution. She had a history of 3 separate reproductive treatments with IVF-ET, but had not become pregnant. An enlarged uterus, similar to that seen after 10 weeks of pregnancy, was noted and MRI findings revealed adenomyosis located on the posterior uterine wall, as well as bilateral endometriotic ovarian 


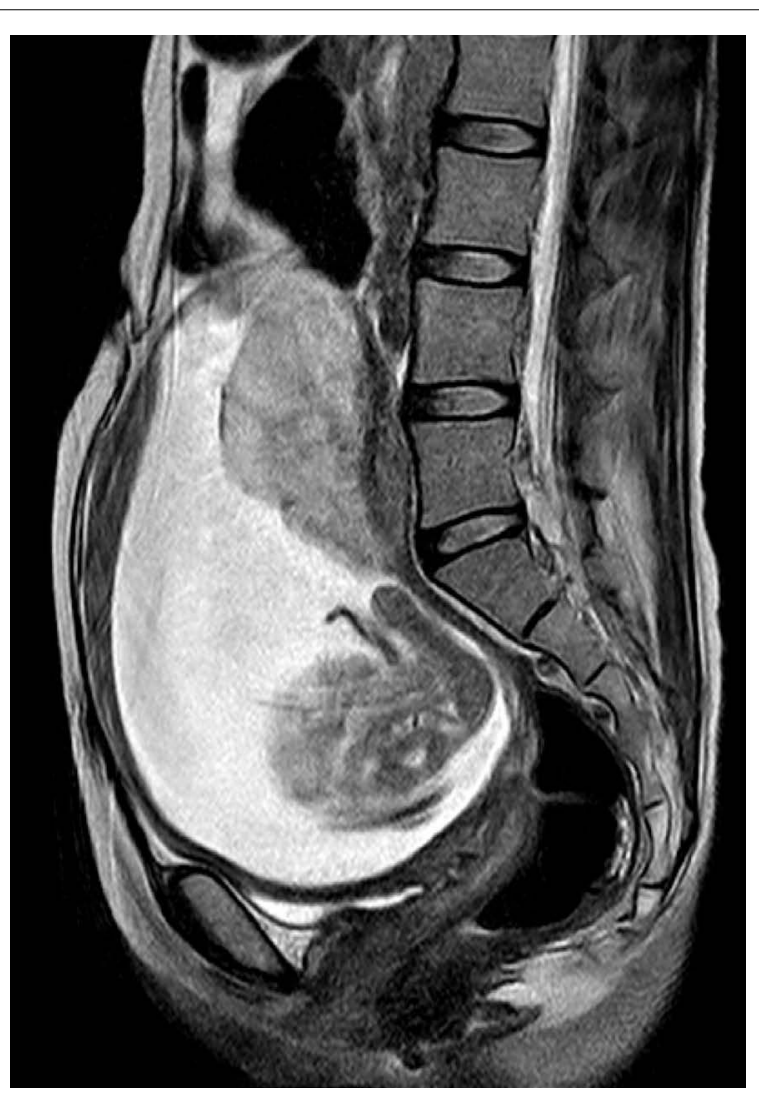

Figure 3: T2-weighted MR images in Case 5 showing sagittal view just before uterine rupture.

Placentation with wave forming and a thin uterine fundal anterior myometrium were observed. Possible placenta percreta was considered from these findings. cysts. We used a Type I procedure and the total weight of the excised adenomyosis tissue was $76 \mathrm{~g}$. Six months after surgery, she conceived with the aid of IVF-ET and achieved a normal pregnancy. At 26 weeks of gestation, the patient was admitted to another institution because of a uterine wall defect shown in ultrasound findings, indicating the possibility of placenta percreta, and was hospitalized. Sudden abdominal pain occurred at 30 weeks of gestation and an emergency caesarean delivery was performed. Laparotomy findings showed that the fundal uterine wall had a longitudinal rupture defect with protrusion of the amniotic sac into the peritoneal cavity, including the fetus and umbilical cord. The fetus weighed $1373 \mathrm{~g}$, had Apgar scores of 5 at 1 minute and 7 at 5 minutes, and survived. The uterine defect was repaired using a classic cesarean procedure. Surgical findings revealed placenta percreta with a uterine rupture.

Case 4: A 34-year-old gravida 0 patient was referred to our hospital in 2011 due to dysmenorrhea with recurrent adenomyosis following a previous adenomyomectomy performed at 31-years-old at another institution. MRI findings revealed adenomyosis lesions involving the entire uterus compatible with diffuse adenomyosis. The surgical method employed was a Type II procedure, and the total weight of the excised adenomyosis tissue was $80 \mathrm{~g}$. Fourteen months after surgery, she spontaneously conceived and achieved pregnancy. However, at 16 weeks of gestation, sudden abdominal pain occurred

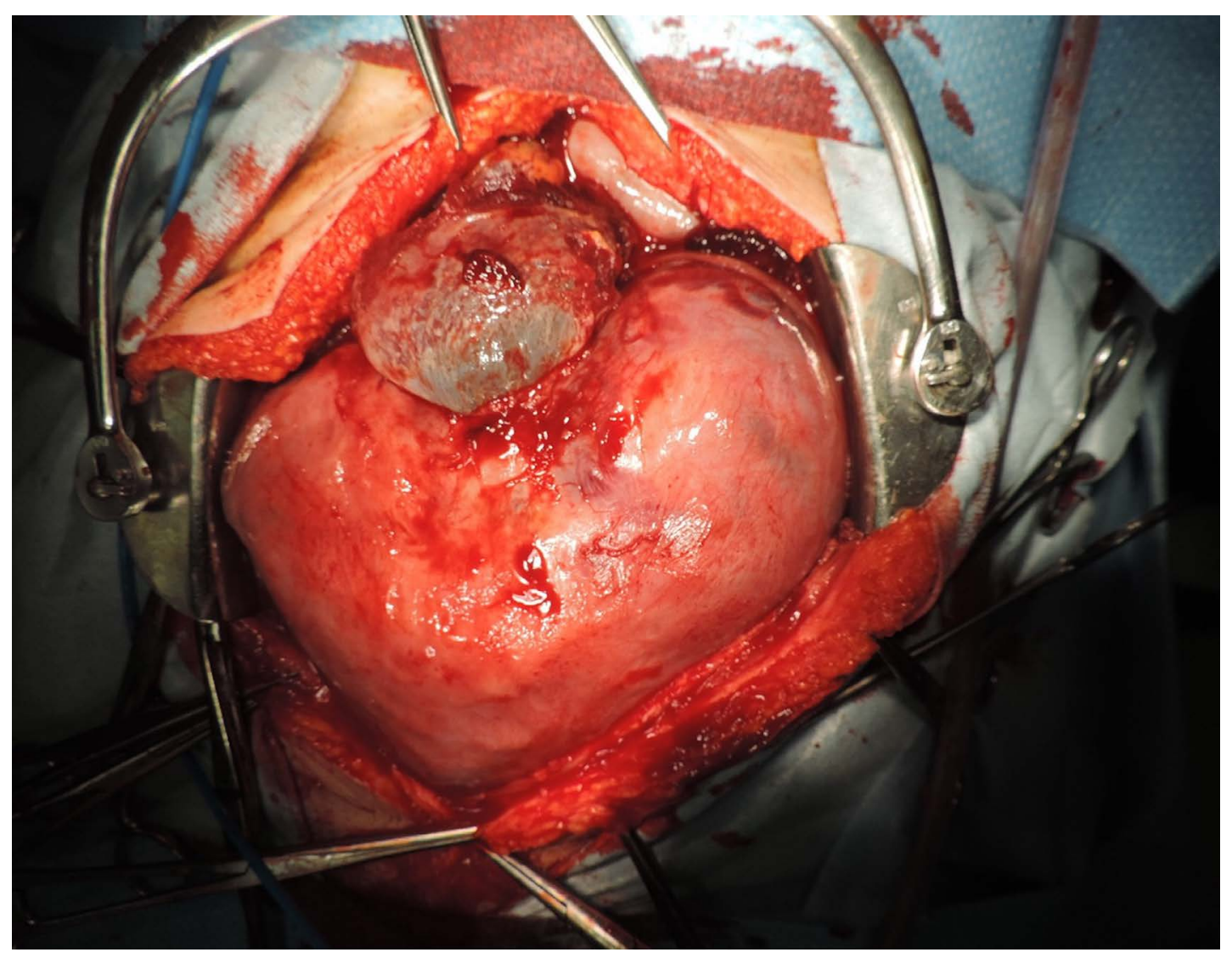

Figure 4: Appearance of uterus in Case 5 when abdomen was opened at uterine rupture.

A protrusion of the amniotic sac and placental tissue were observed. 
requiring an immediate laparotomy performed at our hospital. The fundal uterine wall was found to be ruptured with protrusion of the amniotic sac, including the dead fetus and umbilical cord, into the peritoneal cavity, as well as protrusion of placenta tissue and bleeding. After removal of placental tissue accompanied by excision of normal uterine muscle, the uterine cavity was closed using a two-layer absorbable suture. A pathological examination revealed the existence of chorionic villi invading uterine muscle tissue.

Case 5: We first examined a 32-year-old gravida 0 in 2012 due to infertility, during which diffuse adenomyosis of the posterior wall was found to be dominant. We chose a Type II procedure, the same as in Case 4, for uterine-sparing surgery. Twelve months later, she conceived with the aid of IVF-ET and achieved a normal pregnancy. At 16 weeks of gestation, the patient was admitted to our hospital because of uterine wall thinness shown in MRI findings, indicating the possibility of placenta percreta and uterine rupture (Figure 3). An intravenous administration of ritodrine hydrochloride was started for tocolysis, then increased up to $3.0 \mathrm{p}$. At 19 weeks of gestation, epigastralgia and severe abdominal pain suddenly occurred, which required an immediate laparotomy. Upon opening the abdomen, we noted that the intraperitoneal cavity was occupied with coagulated blood, and a rupture defect with protrusion of the amniotic sac and placental tissue were seen on the fundal uterine wall (Figure 4). After removal of that placental tissue and excision of normal uterine muscle, the uterine cavity was closed using an absorbable suture. The wounded uterus was preserved.

\section{Procedure for adenomyomectomy}

We performed a Type I procedure in Case 1, 2 and 3 for focal adenomyosis, and a Type II procedure in Case 4 and 5 for diffuse adenomyosis. In all of those cases, the uterine cavity was opened and the adenomyosis lesion excised, after which the cavity and myometrium were closed using an absorbable suture. In case 1 and 2 , the uterine myometrium received a Y-shaped suture (Figure 2). In Case 1, 2, and 3, the uterine myometrium was closed using a double layered absorbable suture, while after excision of the adenomyosis lesion in Case 4 and 5 , the uterine cavity was closed using an absorbable suture, followed by uterine rejoining with the outer uterine serosal side covering the inner uterine side with an absorbable suture. In both Case 4 and 5, excision of serosal myometrium on the uterine outer side was performed to a thickness of $2 \mathrm{~mm}$ because of advanced diffuse adenomyosis.

\section{Location of uterine rupture and placentation}

In all cases, the uterine rupture lesion occurred on suture mark of the myometrium and uterine cavity, while placentation occurred in accordance with the excised adenomyosis lesion.

\section{Period of uterine rupture}

Uterine rupture in the 5 cases occurred at 16, 19, 27, 30 , and 31 weeks of gestation, respectively. When such rupture occurred at 27 gestational weeks or later, the neonates survived. On the other hand, when uterine rupture occurred at 16 or 19 weeks of gestation, the fetus was dead, though the patient was able to preserve their uterus.

\section{Symptoms of uterine rupture}

In all 5 cases, sudden abdominal pain occurred with-

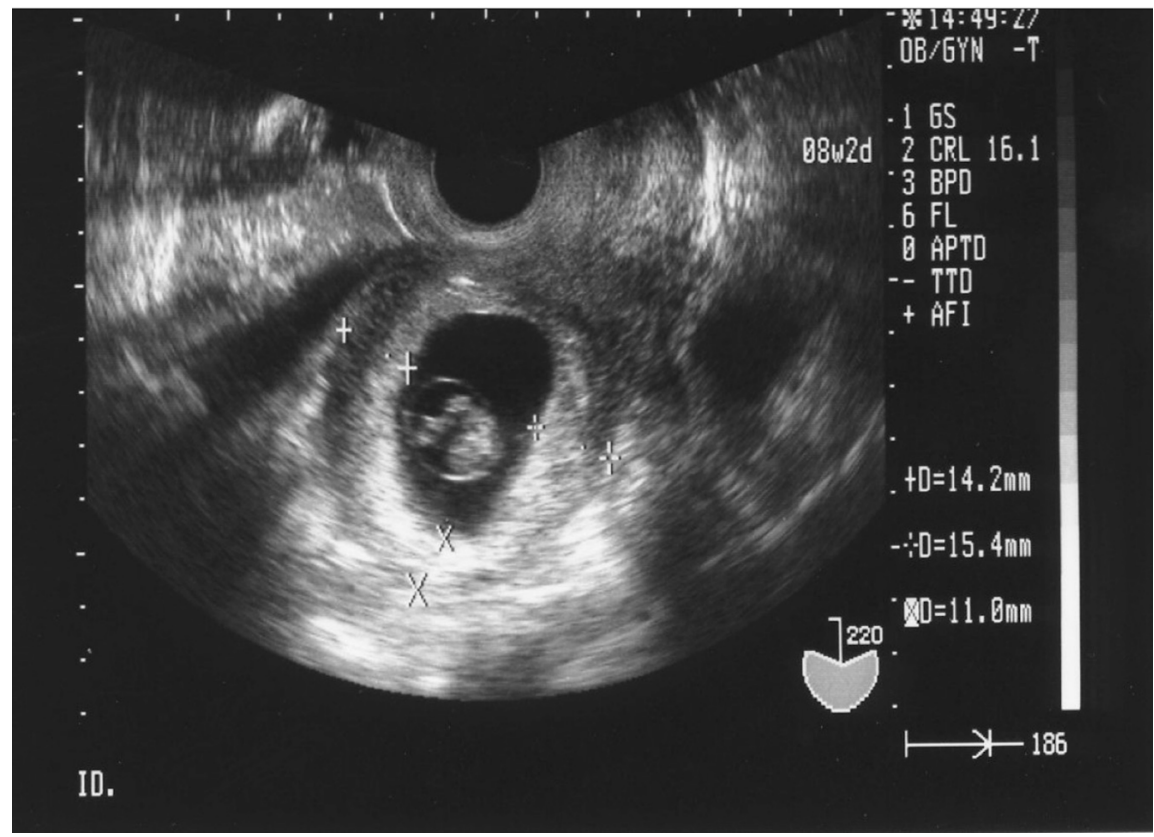

Figure 5: Ultrasound images obtained in early pregnancy in Case 4.

The uterine wall thickness including endometrium and chorionic villi measurements were obtained around $10 \mathrm{~mm}$. 
out perceived uterine contractions. The Case 1, 2, and 5 patients were hospitalized and managed due to abnormal imaging findings suggesting placenta percreta.

\section{Findings during surgery for uterine rupture}

The main findings at the time of laparotomy was bleeding from the abrasion area in the uterus, caused by tearing of the uterine myometrium in all 5 cases. In Case 3 and 4, the uterus had already ruptured, and the fetus was protruding from the uterus into the abdominal cavity, along with the covering amniotic sac and amniotic fluid. In addition, for Case 5, we assumed that the uterine wall would soon become torn, with protrusion of the amniotic sac and placental tissue. Furthermore, pathological examinations revealed chorionic villi in resected myometrium specimens obtained from Case 4 and 5 .

\section{Discussion}

The present cases are shown in chronological order based on the time of uterine rupture. Up to our experience with Case 3, we considered that uterine rupture following an adenomyomectomy occurred in women who underwent a Type I but not a Type II procedure. In our patients, lesions related to uterine rupture occurred on the suture mark of a previous adenomyomectomy procedure, 2 of which had a Y-shaped suture, thus we also considered that such rupture can be caused by a suture-induced imperfection in the uterine wall, or by thinning of the reconstructed uterine wall caused by expansion due to pregnancy and related internal pressure [3,9-11]. In Case 2, the period between surgery and pregnancy was short, even though contraception for 3 months was recommended. However, following our experiences with Case 4 and 5, our ideas regarding these issues have changed.

In Case 4, uterine wall thickness including endometrium and chorionic villi was maintained at around 10 $\mathrm{mm}$ and no remarkable thinness was observed during the early pregnancy period (Figure 5). In contrast, MRI revealed evidence of suspicious placenta percreta just prior to the uterine rupture [12], after which a pathological examination revealed invasion of placental tissue (villi) into the myometrium with residual adenomyosis, which was compatible with a diagnosis of placenta percreta, as seen in Case 5 . We then reviewed our records for Case 1, 2, and 3. In Case 1, a caesarean hysterectomy was performed due to placenta percreta, while the Case 3 patient also received hospitalization due to suspected placenta percreta. Furthermore, in all 5 of the present cases, the common initial symptom of uterine rupture was acute abdominal pain without uterine contraction. Four cases were confirmed to be placenta percreta and intraabdominal bleeding causing abdominal pain was noted during the operation. These findings suggested that the acute abdominal situation during pregnancy following an adenomyomectomy in our patients was caused by placenta percreta.
It is common for a uterine scar rupture to occur with uterine contractions as well as labor, though such a rupture cannot be predicted, only diagnosed after occurrence. When all of the uterine layers are ruptured, referred to as complete or catastrophic rupture, the affected patient will feel a sharp pain. On the other hand, when only stretching or a partially opening occurs, referred to as a 'window' or uterine dehiscence, the condition is not as serious, because the outer layer of the uterus, or perimetrium, remains intact. However, catastrophic rupture and uterine dehiscence are unlikely to occur without a uterine contraction. Therefore, we consider that the trigger for uterine rupture during pregnancy following an adenomyomectomy might be intraperitoneal bleeding resulting from placenta percreta, which cause the placenta to pass through the uterine myometrium above the adenomyomectomy scar. Placenta percreta with uterine contractions causes collapse of a weak uterine myometrium, with bleeding from the broken part occurring thereafter. The time to uterine rupture after the start of bleeding may be relatively long, though intact fetal survival was obtained in Case 1-3. If that time is short, the chance for intact fetal survival is low. We think that a uterine rupture following an adenomyomectomy is not related to the vulnerability of the suture used for uterine reconstruction, but rather placenta percreta. It is possible that placental tissue grows to expand around the suture where the myometrium has been damaged by the adenomyomectomy and then the placenta disrupts uterine myometrium, though the mechanism for placental invasion of the uterine myometrium is unclear.

It is important to pay attention to uterine wall thickness following an adenomyomectomy, as it increases risk for uterine rupture during pregnancy. For non-pregnant patients in whom the uterine wall is observed to be thin, many obstetricians currently recommend contraception or careful management of pregnancy for fear of uterine rupture. The uterus is indispensable for development of a fertilized ovum, though it has not been confirmed that the uterine myometrium is necessary to maintain the pregnancy. Actually, we think that the amniochorionic membrane is vital for maintaining pregnancy. In support of our speculation, once an abdominal pregnancy has been established, clinicians when performing a repeated caesarian section procedure often view the fetus through the uterine myometrium (perimetrium) without rupture. In other words, the role of the uterine myometrium may be only to cover the amniochorionic sac, then provide an expulsive force after conception. Therefore, choice of a cesarean delivery and control of uterine contractions during pregnancy are not related to uterine wall thickness. Effective tocolysis will prevent a uterine rupture regardless of the thickness of the uterine myometrium. Should the amniochorionic membrane remain intact and the uterus have no contractions, the existence of bleeding from placenta percreta tissue is related to prognosis. 


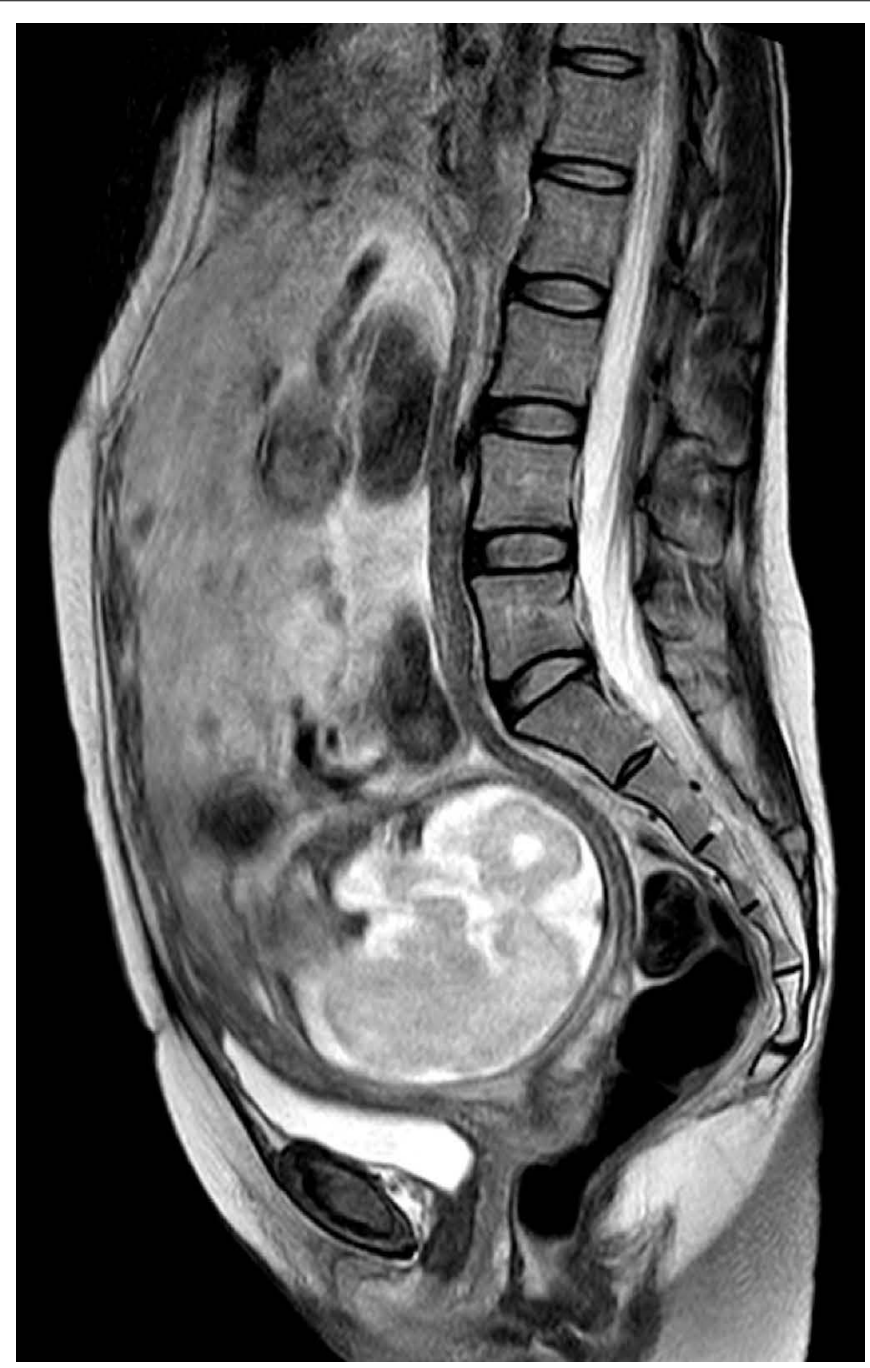

Figure 6: T2-weighted MR images showing sagittal views at 30 weeks of gestation.

We observed that the upper anterior segment of the uterine myometrium was divided by placental tissue as well as flow void. These findings confirmed a diagnosis of placenta percreta.

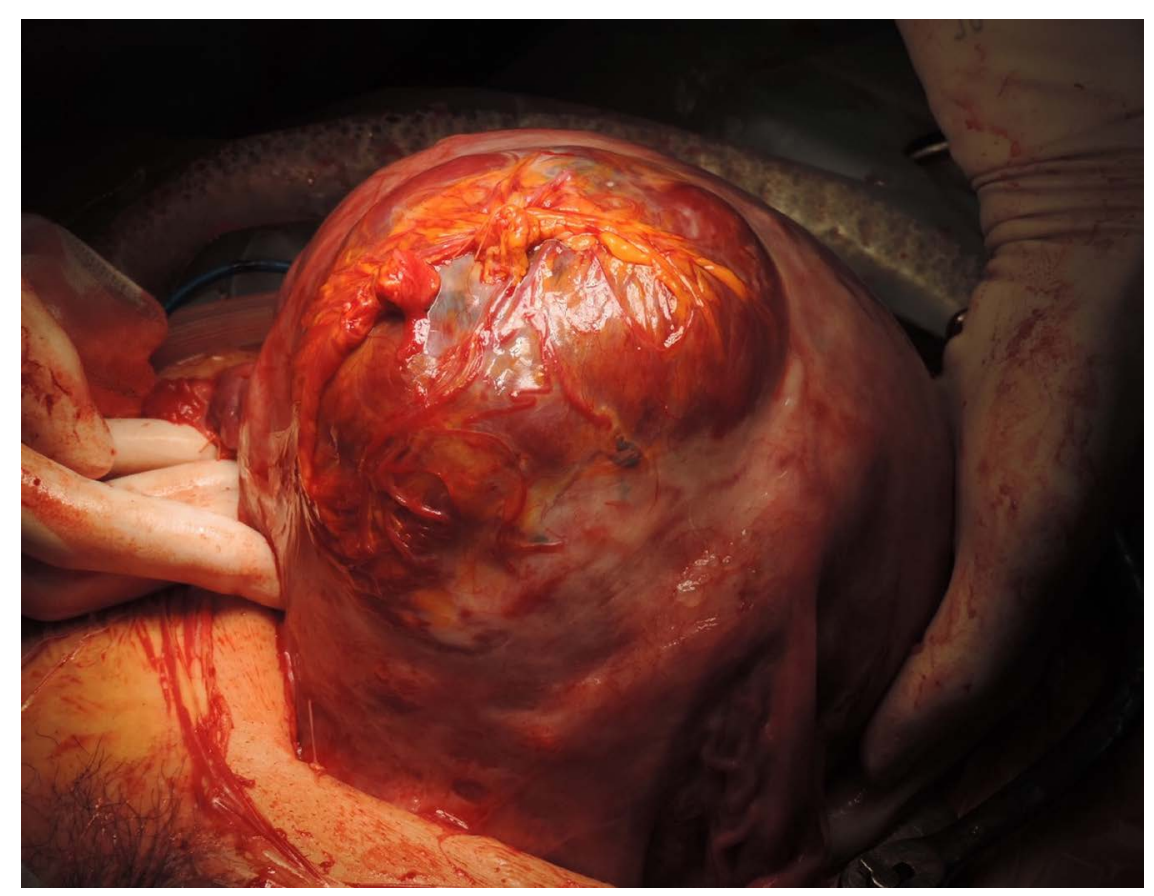

Figure 7: Appearance of uterus when abdomen was opened.

In the upper anterior segment of the uterine myometrium, placental tissue covering the uterine serosa along with adhesion to the greater omentum were observed. 
Based on consideration of these issue, we hereby suggest a proposal for prevention of uterine rupture following an adenomyomectomy. Once uterine dehiscence is diagnosed, it should be treated with tocolysis agents for an appropriate period. Regardless of placentation, prevention of intrapartum rupture of the scarred uterus is dependent on such treatment. Next, it is important to confirm the suture mark on the uterine myometrium and its location in relations to the placenta. There is a low risk of uterine rupture if placentation is not on the suture mark from which the adenomyosis was excised. On the other hand, additional examinations, such as color Doppler ultrasonography and MRI, should be performed to exclude placenta myometrium invasion when placentation is on the suture mark. When a case is diagnosed as placental adherence, there are 2 techniques available, depending on the degree. One method is to treat the condition with a tocolysis agent for an appropriate period to allow for intact fetal survival [13] and also prevent symptoms of uterine rupture, though a clinician must be available at all times for a potential emergency cesarean delivery. The other method is to take precautions to prevent bleeding [14] from the area of possible placenta increta or percreta, because a uterine rupture will not develop without a broken uterine perimetrium caused by placenta percreta and bleeding.

Following are details for 2 of our successful cases that did not experience uterine rupture during pregnancy. Figure 6 presents T2-weighted MR images showing sagittal views at 30 weeks of gestation, when a defini- tive diagnosis was made. This patient was hospitalized because of possible uterine rupture and treated with an intravenous administration of ritodrine hydrochloride for tocolysis until performance of a scheduled cesarean section at 35 weeks of gestation. Figure 7 shows the appearance of the uterus when the abdomen was opened. Placental tissue covering the uterine serosa along with adhesion to the greater omentum were observed, while no bleeding that results to acute abdomen was observed at the perimetrium. Figure 8 shows the appearance of the uterine fundus in another case at 15 weeks of gestation, for which Tachosil $^{\circledR}$ was administered because of possible placenta percreta. This patient successfully continued the pregnancy until 35 weeks of gestation without uterine rupture.

Based on our findings, some mechanisms of uterine rupture after an adenomyomectomy are suggested as well as possible surgical/therapeutic strategies to prevent a future rupture in affected patients. Although we consider that our suggestions are theoretically reasonable, our considerations are drawn from experiences only 5 patients. Thus, no definite conclusions regarding these issues can be made and additional investigations are needed.

\section{Conclusion}

We began providing treatment for adenomyosis to respond to our patients wishing for relief from severe pain and menorrhagia. Patients for whom surgery is required often assume that infertility will be the result, though some of our mild asymptomatic patients have

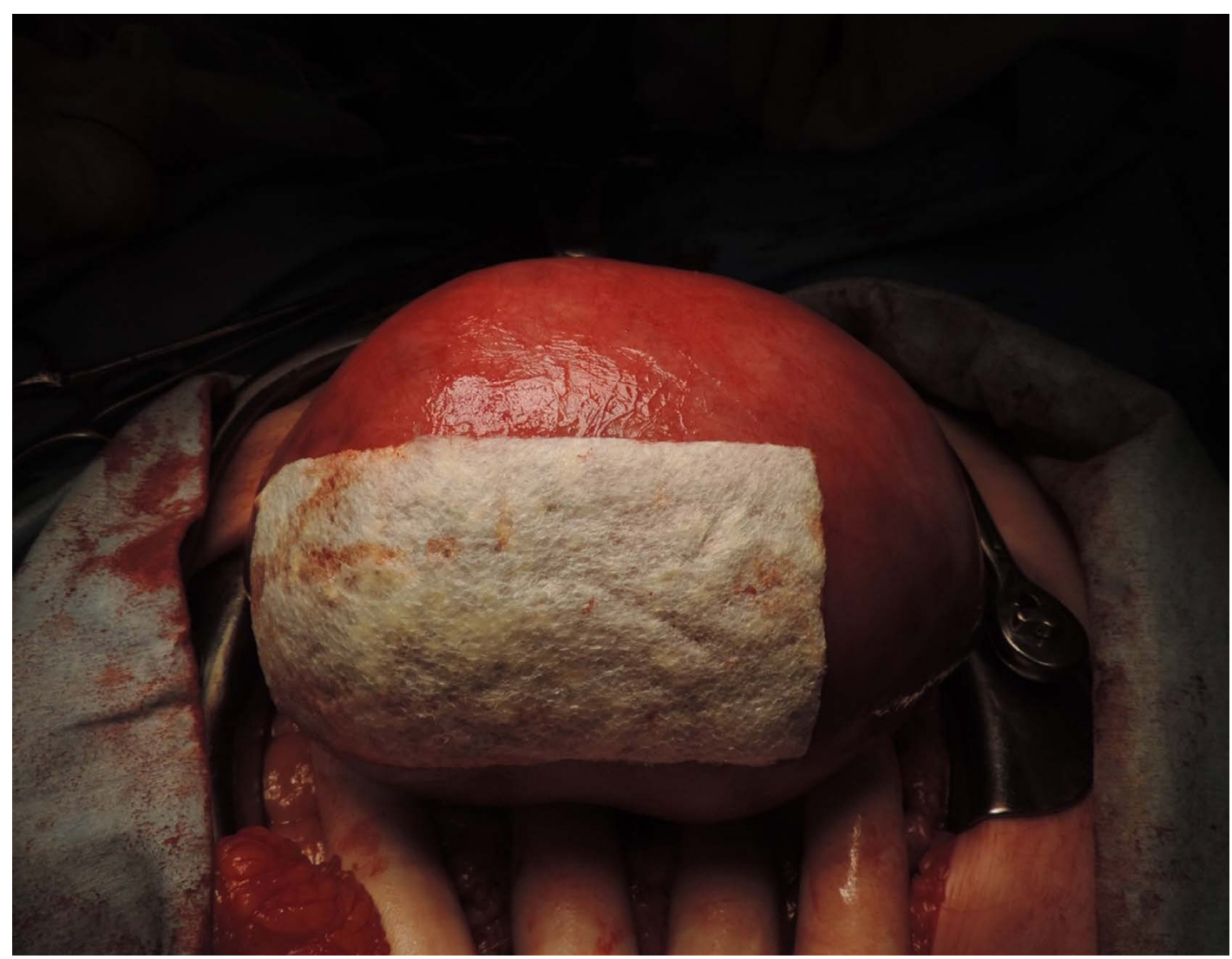

Figure 8: Appearance of the uterine fundus covered with Tachosil ${ }^{\circledR}$ at 15 weeks of gestation. 
become pregnant and delivered. Our goal is that women treated with our procedure are able to conceive, though the risk of uterine rupture is a major concern. Therefore, we believe that the present findings will be useful for obstetric managements of patients who become pregnant following an adenomyomectomy procedure.

\section{Acknowledgement}

This study was approved by the Institutional Ethical Committee (permission No. 26-10) on March 2015, and informed consent was obtained from each of the patients.

\section{Conflict of Interest}

The authors declare that they have no conflict of interest.

\section{Authors Contribution}

M Nishida: Project development, Data collection, Manuscript writing.

Y Otsubo: Data collection, Manuscript writing.

Y Arai: Data collection, Manuscript editing.

R Ichikawa: Data collection.

Y Kondo: Data collection, Pathological analysis.

H Itagaki: Data collection.

M Sakanaka: Data collection.

\section{References}

1. Leyendecker G, Kunz G, Kissler S, Wildt L (2006) Adenomyosis and reproduction. Best Pract Res Clin Obstet Gynecol 20: 523-546.

2. Nishida M, Takano K, Arai Y, Ozone H, Ichikawa R (2010) Conservative surgical management for diffuse uterine adenomyosis. Fertil Steril 94: 715-719.
3. Otsubo $Y$, Nishida M, Arai $Y$, Ichikawa R, Taneichi A, et al. (2016) Association of uterine wall thickness with pregnancy outcome following uterine-sparing surgery for diffuse adenomyosis. Aust NZJ Obstet Gynaecol 56: 88-91.

4. Wada S, Kudo M, Minakami H (2006) Spontaneous uterine rupture of a twin pregnancy after a laparoscopic adenomyomectomy: A case report. J Minim Invasive Gynecol 13: $166-168$.

5. Keifer WS (1964) Rupture of the uterus. Am J Obstet Gynecol 89: 335-348.

6. Kishi $Y$, Suginami H, Kuramori R, Yabuta M, Suginami R, et al. (2012) Four subtypes of adenomyosis assessed by magnetic resonance imaging and their specification. Am J Obstet Gynecol 207: 114.e1-114.e7.

7. Farquhar C, Brosens I (2006) Medical and surgical management of adenomyosis. Best Pract Res Clin Obstet Gynecol 20: 603-616.

8. Nishida M, Okamoto H, Someya K, Kubo T (1996) Metroplasty for the treatment of unicornuate uterus. J Gynecol Tech 2: 89-94.

9. Seinera P, Farna C, Todros T (2000) Laparoscopic myomectomy and subsequent pregnancy: Results in 54 patients. Hum Reprod 15: 1993-1996.

10. Yazawa H, Endo S, Hayashi S, Suzuki S, Ito A, et al. (2011) Spontaneous uterine rupture in the 33rd week of IVF pregnancy after laparoscopically assisted enucleation of uterine adenomatoid tumor. J Obstet Gynaecol Res 37: 452-457.

11. Grimbizis G, Mikos T, Tarlatzis B (2014) Uterus-sparing operative treatment for adenomyosis. Fertil Steril 101: 472-487.

12. Masselli G, Gualdi G (2013) MR imaging of the placenta: What a radiologist should know. Abdom Imaging 38: 573-587.

13. Otsubo Y, Nishida M, Arai Y, Ichikawa R (2016) Spontaneous conception and two successful pregnancies in patient who underwent conservative surgical management of diffuse uterine adenomyosis. J Obstet Gynaecol 36: 830-832.

14. Sugawara T, Obawa M, Tanaka T (2014) Repair of uterine rupture during second trimester leading to successful pregnancy outcome: Case study and literature's review. AJP Rep 4: 9-12. 